\title{
Elementos físico-químicos contaminantes presentes en el arroyo Cañafístula del municipio de Polonuevo, Atlántico
}

\section{Physicochemical contaminants elements present in the Cañafístula stream in the municipality of Polonuevo, Atlántico}

\author{
Yurelis Cáceres ${ }^{*}$, Juan Carlos Arrieta Ruiz ${ }^{* * *}$ y Jairo Enrique Salazar Barrios" ${ }^{* * * * *}$
}

Citar este artículo como: Cáceres, Y.; Arrieta Ruiz, J. C. y Salazar Barrios, J. E. (2018). Elementos fisicoquímicos contaminantes presentes en el arroyo Cañafístula del municipio de Polonuevo, Atlántico. Revista Nodo, 12(24), 60-73.

\section{Resumen}

La preocupación global con respecto al manejo del agua es producto de la crisis ambiental que posee el mundo moderno, siendo el agua uno de los elementos constituyentes de la vida y de los seres vivos. Colombia no es ajeno a esta situación, ya que al ser un país con una alta riqueza hídrica, no le exime de ser contaminada por diversos agentes fisicoquímicos y biológicos, los cuales podrían ocasionar daño a los ecosistemas donde se encuentran, llevándole a perder paulatinamente la calidad de vida de los mismos, razón que motivó la investigación donde se buscó identificar los elementos fisicoquímicos contaminantes presentes en el arroyo Cañafístula del municipio de Polonuevo, Atlántico.

El estudio se llevó a cabo empleando un tipo de investigación descriptiva con enfoque mixto bajo un paradigma hermenéutico-interpretativo. Se utilizó como técnica de recolección de la información la observación y como instrumentos las herramientas de laboratorio para análisis y toma de muestras. Se ubicaron puntos de recolección a lo largo del arroyo Cañafistula del municipio de Polonuevo, colocados estratégicamente desde el inicio del arroyo hasta el final. Los resultados evidenciaron que existen agentes fisicoquímicos fuera de los parámetros permitidos en la norma producto de los residentes del municipio y de las granjas que le rodean, sin evidenciar niveles de contaminación preocupantes que al no ser tratados oportunamente llegarían a afectar la población.

Palabras clave: Contaminación, Aguas residuales, arroyos, vertimientos, Demanda química de oxígeno (DQO), Demanda bioquímica de oxígeno (DBO5)

Fecha de recibo: 4 de octubre de 2017 • Fecha de aceptación: 20 de julio de 2018

\footnotetext{
* Magister en Ciencias Ambientales de la Universidad del Atlántico- SUE Caribe, Grupo de Investigación CETIC, Barranquilla, Atlántico. Correo electrónico: yurelis0711@hotmail.com

* Docente de la Universidad del Atlántico, Coordinador del Programa de Licenciatura en Biología y Química, Grupo de Investigación CETIC, Barranquilla, Atlántico. Correo electrónico: juanarrieta@mail.uniatlantico.edu.co

*** Docente de la Universidad del Atlántico, Grupo de Investigación CETIC, Barranquilla, Atlántico. Correo electrónico: jairosalazar@mail.uniatlantico.edu.co
} 


\section{Abstract}

Global concern about the management of water and its sources is product of the environmental crisis of the modern world, since water is one of the constituent elements of life and living beings. Colombia is no stranger to such concern, since being a country with a great water wealth, this does not exempt it from being contaminated by various physicochemical and biological factors, which could cause harm not only to humans but also to ecosystems where they are, which can lead to gradually losing their quality of life. For this reason, the present research sought to identify the physicochemical contaminants elements present in the Cañafístula stream of the municipality of Polonuevo, Atlantic.

The study was carried out using a type of descriptive research with a mixed approach under an interpretive hermeneutic paradigm. Observations were used as a technique for collecting the information and the laboratory tools for analysis and sampling. The sample consisted of sampling points from the Cañafistula stream in the municipality of Polonuevo, strategically located from the beginning of the stream to the end. The results showed that there are physicochemical agents outside the parameters allowed in the product standard, produced by the residents of the municipality and of the farms that surround it, without evidencing contaminating levels of contamination, when not evidencing that treated in a timely manner, would affect the population.

Keywords: Contamination, Wastewater, Streams, dumping, Chemical oxygen demand (COD), Biochemical Oxygen demand (BOD5)

Una de las fuentes principales de contaminación es el crecimiento demográfico, al generar mayor cantidad de aguas residuales.

\section{Introducción}

En 2016, el Programa de las Naciones Unidas para el Medio Ambiente puso en evidencia la problemática mundial relacionada con las fuentes hídricas, principalmente en los continentes de Asia, África y América Latina, advirtiendo sobre un posible desastre ambiental en los países pertenecientes a estos, el cual se vería reflejado en la salud y la política de sus habitantes ocasionando eco a nivel global (PNUMA, 2016).

El agua es uno de los recursos naturales esenciales para la vida del planeta mismo, y al ser modificado por las diversas formas de contaminación a la que se enfrenta, origina alteración del entorno natural de los ecosistemas, ocasionando afectaciones significativas en la calidad de vida los seres vivos, incidiendo en el bienestar de los mismos, produciendo enfermedades relacionadas con el agua. Y a su vez, afectando la economía directa de los pueblos debido a la inversión que debe hacerse no solo para la salud sino también en el tratamiento de la contaminación (PNUMA, 2004).

Una de las fuentes principales de contaminación es el crecimiento demográfico, al generar mayor cantidad de aguas residuales; sobre este aspecto la Organización de las Naciones Unidas para la Educación, la Ciencia y la Cultura UNESCO (2016), afirma que la raza humana ha aumentado sustancialmente tanto que en el 2016 el planeta albergaba aproximadamente 7 billones de habitantes y se calcula que para el 2050 este número alcanzaría unos 9.5 billones, originando un crecimiento urbanístico, en especial en paises en via de desarrollo, lo que produciría una mayor descarga de aguas residuales al medio ambiente.

El vertimiento de las aguas residuales ocasiona cambios en los elementos propios de la fuente hídrica, tanto en aspectos fisicoquímicos como microbiológicos. La legislación Colombiana define aguas residuales como: "Agua que no tiene valor inmediato para el fin para el que se utilizó ni para el propósito para el que se produjo debido a su calidad, cantidad o al momento en que se dispone de ella." (FAO, 2015). 
Teniendo en cuenta el origen y los cantaminates que contiene Espigares y Pérez (2003), clasifican las aguas residuales en:

- Aguas Negras, producto de actividades domésticas del ser humano y contenedoras de productos de limpieza, heces fecales, orina entre otros;

- Blancas, las originadas por las lluvias o de la limpieza de lugares públicos; industriales y agrícolas, producto de residuos de procesos químicos o derivados de la agricultura y que pueden poseer alcoholes, detergentes, grasas, hidrocarburos, plaguicidas, abono químicos entre otros.

Las aguas residuales modifican los aspectos físicos naturales como son: el color, la turbidez, la temperatura, sedimentos, olor y sólidos, éstos pueden ser orgánicos e inorgánicos. A su vez modifica aspectos químicos como son: los gases disueltos, entre ellos el oxígeno disuelto; potencial de óxidoreducción; conductividad; $\mathrm{pH}$ y salinidad.

Diversos autores han indagado acerca del efecto de las aguas residuales en los aspectos fisicoquímicos del las fuentes hídricas; entre ellos se destaca a nivel internacional el trabajo de Ahmed et al (2016) para la Universidad de Queensland (Australia), cuyo objetivo fue determinar el nivel de contaminación fecal humana en aguas superficiales australianas ubicadas en diferentes zonas climáticas del país, mediante ensayos PCR cuantitativos, hallando contaminación por E. Coli, HF183 y Esteptococcus.

Núñez (2015) desarrolló su investigación con el propósito de conocer cuáles son los efectos negativos del uso de aguas residuales en Ixmiquilpan Hidalgo (México). Utilizó una metodología mixta ya que se hizo una vinculación y análisis de lo que son los métodos cualitativos y cuantitativos. Como resultado de este estudio, se encontró que los problemas derivados de las aguas residuales presentaron afectaciones en la salud humana, debido al contenido de microorganismos patógenos tales como bacterias, virus, parásitos, y contaminantes químicos, etc., que son vertidos llegando a provocar enfermedades como cólera, diarreas o afectaciones en la piel por el contacto y consumo de aguas contaminadas o por el consumo de vegetales que son regados con estas.

Rosedo (2014), en su estudio sobre el impacto y manejo ambiental producto de la operación y mantenimiento de la planta de tratamiento de aguas residuales del barrio La Primavera de la parroquia Yanayacu del cantón Quero, provincia de Tungurahu, utilizó para la recolección de información encuestas, entrevistas y observaciones dentro de las áreas de influencia, además de la toma de muestras para análisis de laboratorio y estadístico. Los resultados arrojaron que los parámetros nitratos, fosfatos, $\mathrm{DBO}_{5}$, aceites y las colonias de coliformes fecales se encuentran sobre el límite máximo permitido en la norma.

A nivel nacional se destaca la investigación de Ramírez (2015) cuyo objetivo fue obtener información de las problemáticas ambientales presentes en diversas regiones de Colombia a partir de la percepción de estudiantes universitarios residentes en dichas zonas. Para el estudio empleó un diseño exploratorio, descriptivo y de corte transversal, en el cual se aplicó un cuestionario vía internet a 429 estudiantes ( $27 \%$ de la población), según los resultados se obtuvo información de 175 municipios del país (16\% del total nacional) donde la principal problemática ambiental identificada fue la contaminación del agua (39.6\%), fundmentalmente debido a la alteración de la calidad del agua de cuerpos superficiales (ríos, quebradas, humedales, lagunas y canales) por efecto de vertimientos líquidos y disposición de residuos sólidos generados en mayor medida por viviendas de uso domiciliario y actividades agrícolas.

Por otro lado, Hidalgo y Mejía (2010) realizaron un estudio cuyo fin fue evaluar la afectación del recurso hídrico por el vertimiento de las aguas residuales domésticas provenientes de descargas directas o de los sistemas de tratamiento integrado en la cuenca baja de la quebrada La Macana, en el corregimiento de San Antonio de Prado, municipio de Medellín, durante el año 2009. Se muestrearon 7 puntos para parámetros indicadores de la calidad $\mathrm{DBO}_{5}, \mathrm{DQO}$, coliformes totales, E. Coli, grasas y 
aceites y sólidos suspendidos y se realizaron entrevistas dirigidas. Los resultados arrojaron que el $62 \%$ de la carga total proviene de las viviendas con tanque séptico y el restante $38 \%$ de las que realizan el vertido directo. La relación $\mathrm{DBO} / \mathrm{DQO}$ muestra que en el tramo estudiado (300 m), la quebrada ha degradado el $80 \%$ de la carga contaminante debido a la alta capacidad de autodepuración de la corriente.

A nivel local se encuentra un informe presentado por el Ministerio de Salud y Protección Social en el año 2014, en relación a la calidad de agua para el consumo humano en el municipio de Polonuevo, en el cual se indica que éste no presenta riesgos. Los resultados analizados por el Índice de Riesgo para la Vigilancia de la Calidad del Agua para Consumo Humano IRCA's reportados por la autoridad sanitaria al Sistema de Información de la SIVICAP, en ejercicio de la vigilancia se llevó a cabo en la mayoría de los municipios durante todo el año y solamente seis lo hicieron durante un promedio de 10 meses. No obstante, la información obtenida del anterior estudio, se contradice con los reportes entregados por la E.S.E. Hospital Local de Polonuevo sobre la prevalencia de enfermedades de origen hídrico por época (seca y lluviosa) y grupos etarios.

Lo que refiere a Colombia, éste es reconocido como uno de los países con una alta disponibilidad del agua (OCDE, 2014), sin embargo no es ajeno a las problemáticas mencionadas, por lo tanto enfrenta un daño ambiental producto de las aguas residuales. En el informe entregado por el Ministerio de Agricultura (2011) se manifiesta que Colombia sólo trata el $23 \%$ de las 1.230 .193 ha. de aguas residuales, dejando un $73 \%$ sin tratar, las que usualmente son diluidas con aguas superficiales. Sin embargo, tal como ocurre en otros países, solo evidencia que el $8 \%$ del total de las aguas son tratadas, ya que no se cuenta con la información completa y confiable que indique realmente el uso y reutilización de este preciado recurso.

El municipio de Polonuevo, ubicado en la margen oriental del departamento del Atlántico no es ajeno a esta situación, a pesar de que sus cuerpos de agua son intermitentes o se forman con las aguas lluvias, se logró identificar problemas como contaminación por desechos sólidos domiciliarios y vertimientos líquidos en el arroyo Cañafístula perteneciente a la microcuenca del Arroyo Grande, que a su vez hace parte de la gran cuenca del río Magdalena (Alcaldía municipal de Polonuevo, 2016).

El arroyo Cañafístula nace en el sector de Lomas, localizado entre las poblaciones de Baranoa y Polonuevo, aproximadamente sobre la cota 120 m.s.n.m. Toma inicialmente un alineamiento de dirección SE hasta llegar a la región de Andrea, en donde toma rumbo NE para dirigirse a la población de Sabanagrande. Los afluentes más importantes son los arroyos San Nicolás y Fernández, pasa por el costado sur de Sabanagrande y desemboca en la ciénaga del mismo nombre. Esta cuenca también se encuentra profundamente transformada con reemplazos completos de sus coberturas originales. Presenta la mejor expresión de la planicie eólica, con las dunas alineadas en sentido NNE. En esta microcuenca se localizan los centros urbanos de Santo Tomás y parte de Sabanagrande y Polonuevo.

Morfológicamente esta cuenca tiene también tres elementos: la zona baja cuyos dominios están marcados por la divagación del río Magdalena con los orillares propios, y las formas de cubeta que permanentemente viven ocupadas por agua; la

\section{En el informe entregado por el Ministerio de Agricultura (2011) se manifiesta que Colombia sólo trata el $23 \%$ de las 1.230 .193 ha. de aguas residuales, dejando un $73 \%$ sin tratar, las que usualmente son diluidas con aguas superficiales.}


zona de terraza aluvial plana con influencia eólica, y un pequeño valle estrecho que se forma en la conjunción del arroyo San Nicolás con el arroyo Cañafístula, hasta su entrega en los complejos cenagosos (Corporación Autónoma Regional del Atlántico, 2005).

Por otro lado, para el estudio del cual da cuenta este artículo tuvo en cuenta la normatividad al respecto del manejo de aguas residuales: Decreto 1076 de 2015; Resolución 0631 de 2015; Decreto 3930 de 2010; Decreto 1323 de 2007; Decreto 3100 de 2003; Ley 812 de 2003; Ley 142 de 1994; Ley 99 de 1993 y Decreto-Ley 2811 de 1974.

\section{Metodología}

El presente estudio es de tipo descriptivo, conocido como estudio transversal, con un enfoque mixto. Su área de estudio fue el municipio de Polonuevo, siendo ésta una de las 23 localidades que conforman el departamento del Atlántico; se encuentra a una distancia de 28 kilómtros de la ciudad de Barranquilla. Limita al norte con el municipio de Malambo, al sur con los municipios de Sabanalarga y Ponedera, al este con el municipio de Santo Tomás y Sabanagrande, y al oeste con el municipio de Baranoa.

En 2016 contaba con una población total, según proyecciones del DANE (2005), de 15.407 habitantes, con un $0,6 \%$ del total de la población del Departamento. De éstos, 12.634 (82\%) habitan en la cabecera y 2.773 (18\%) en el resto del área de Polonuevo. A solo 33 kilómetros de la ciudad de Barranquilla por la vía la cordialidad y 40 kilómetros por la vía oriental. Y tiene una cabida superficial de 75,14 Km2 que representa el 2,29\% del total del departamento del Atlántico, de los cuales, $1,10 \mathrm{~km} 2$ lo ocupa la cabecera municipal y $74,04 \mathrm{~km} 2$ el área rural.

Esta cabecera urbana se encuentra ubicada dentro del contexto del departamento en la cuenca hidrográfica del río Magdalena, la cual está conformada por las subcuencas de los humedales del río Magdalena, que drenan por toda la parte oriental del departamento de sur a norte desde el límite con Bolívar hasta Bocas de Cenizas, cuenta con una superficie de 134.192 hectáreas. Con el arroyo Cañafistula como parte del recurso hídrico del municipio, cuya longitud es de $6 \mathrm{~km}$ y un área de 60ha.

El estudio se realizó mediante la toma de muestras en diferentes puntos a lo largo del arroyo Cañafistula, empleando las normas establecidas en el Standard Methods for the Examination of Water and Wastewater (APHA, 2005). Se establecieron 2 estaciones georeferenciadas en el arroyo, para los que se tuvo en cuenta la distribución y ubicación para extraer el agua para ser analizada que permitieran obtener información de todo el recorrido de los arroyos. La obtención se realizó utilizando una botella de vidrio ámbar, esterilizada, con capacidad para 1 litro, se llenó la botella con el líquido, se cerró, refrigeró y procedió al laboratorio para ser examinada.

Análisis de las muestras: la metodología usada para el análisis de las muestras se basa en procedimientos establecidos por la Environmental Protection Agency (EPA), estandarizados, validados e implementados en los laboratorios de la Universidad del Atlántico, de la Corporación Universitaria de la Costa, además del laboratorio Labomar.

Para analizar la concentración de residuos orgánicos en muestras de aguas superficiales se determinó inicialmente la Demanda Química de Oxígeno a través del método Colorimétrico de reflujo cerrado (SM 5220D) aplicable en aguas naturales y residuales domésticas y no domésticas. Es un parámetro que mide la cantidad de sustancias susceptibles de ser oxidadas por medios químicos, que hay disueltas o en suspensión en una muestra líquida. Se utiliza para medir el grado de contaminación y se expresa en mg O2/L.

La evaluación de la DQO es sugerida dentro de los análisis de aguas, ya que sirve inicialmente como base para la estimación de la DBO. La DQO es un método definido; el grado de oxidación de la muestra puede estar afectada por el tiempo de digestión, la concentración de los reactivos y la concentración de 
DQO en la muestra. Para el método colorimétrico se cuenta con viales listos para su uso; la lectura realizada por un fotómetro o un espectrofotómetro (Universidad de la Guajira, 2016).

También se estimó la demanda bioquímica de oxígeno, DBO5, en aguas; este análisis se requiere en fuentes hídricas que han podido ser contaminadas por aguas residuales para valorar los efectos de las descargas de los efluentes domésticos y no domésticos sobre la calidad de los cuerpos receptores. Básicamente es una medida de la cantidad de oxígeno requerido para degradar la materia orgánica de una muestra de agua, por medio de una población microbiana heterogénea. La información obtenida en la prueba corresponde a la materia orgánica biodegradable.

El procedimiento estandarizado del laboratorio es usado para determinar los requerimientos relativos en el agua residual, efluentes o aguas contaminadas. El análisis mide el oxígeno molecular utilizado durante un período especificado de incubación para la degradación bioquímica de la materia orgánica (demanda carbonácea) y el oxígeno utilizado para oxidar la materia inorgánica (hierro ferroso y sulfuros) presente en una muestra de agua. También puede medir el oxígeno utilizado para oxidar formas reducidas del nitrógeno (demanda nitrogenácea) a menos que se prevenga esta oxidación por medio de un inhibidor.

Los resultados fueron analizados con ayuda de estadística descriptiva, con la finalidad de organizar la información referente a los análisis fisicoquímicos realizados en cada punto de monitoreo. Adicionalmente, se estableció el coeficiente de Correlación de Pearson para determinar la incidencia de los niveles de contaminación en los arroyos Cañafistula y Pica Pica. Para todos los análisis estadísticos el criterio de significancia se estableció con un p<0,05.

\section{Resultados y discusión}

Los resultados obtenidos después de analizar las muestras de aguas obtenidas de los distintos puntos asignados en el los arroyo Cañafístula del municipio de Polonuevo (Atlántico) arrojaron que las principales fuentes de contaminación son producto de las costumbres alimentarias de los moradores, el vertimiento de las aguas residuales domésticas, la carencia de sistema alcantarillado municipal y las actividades agrícolas y campesinas de la población, las cuales ocasionan variación en los valores de los parámetros físico-químicos, reflejados en la tabla 1.

Tabla 1. Caracterización fisicoquímica de las aguas del arroyo Cañafístula.

\begin{tabular}{|l|l|}
\hline Turbiedad & \multicolumn{1}{|c|}{$\begin{array}{c}\text { Rango Arroyo } \\
\text { Cañafístula }\end{array}$} \\
\hline Temperatura & $15,95-23,15 \mathrm{UNT}$ \\
\hline Conductividad & $30,40-34,50{ }^{\circ} \mathrm{C}$ \\
\hline Solidos totales disueltos & $0,59-1,01 \mathrm{uS} / \mathrm{cm}$ \\
\hline DQO & $0,38-1,01 \mathrm{mg} / \mathrm{L}$ \\
\hline DBO $_{5}$ & $36,92-86,60 \mathrm{mg} / \mathrm{L}$ \\
\hline pH $_{\text {Salinidad }}$ & $3,20-16,00 \mathrm{mg} / \mathrm{L}$ \\
\hline Oxígeno disuelto & $6,18-7,96$ \\
\hline Potencial de óxido reducción & $0,20-0,63 \mathrm{mg} / \mathrm{L}$ \\
\hline
\end{tabular}

Fuente: elaboración propia

Las muestras analizadas, presentaron una coloración gris, olor desagradable y aspecto turbio $(15,95$ - 23,15 UNT), sin presencia de material flotante. El color y el olor detectados son características típicas de las aguas residuales municipales frescas y oxigenadas. La caracterización de las aguas del arroyo demostraron que los parámetros ambientales temperatura, $\mathrm{pH}$ y conductividad no son contaminantes por encontrarse los valores determinados por debajo del límite máximo permisible que regula el Ministerio de Medio Ambiente (2015).

Parámetros físicos: Se determinaron los parámetros físicos tales como temperatura, turbiedad, conductividad y cantidad de sólidos totales disueltos, encontrándose los siguientes resultados: 
Temperatura. De acuerdo con informes del Instituto de Hidrología, Meteorología y Estudios Ambientales (IDEAM, 2016), durante los primeros seis meses de 1995 las diferentes estaciones registraron precipitaciones así: $615,4 \mathrm{~mm}$ la Normal de Manatí; 380,6 mm la del Limón; 325,6 mm la de Repelón y 164,7 mm la del Aeropuerto Ernesto Cortissoz. Teniendo en cuenta esas variables, el clima del Atlántico hace parte de los climas tropicales secos de tipo estepario con altas temperaturas.

Durante los monitoreo de calidad efectuados en la zona de estudio se registraron temperaturas que oscilaron entre $25^{\circ} \mathrm{C}$ y $37^{\circ} \mathrm{C}$. Los menores valores de temperatura correspondieron a los observados durante los monitoreos en condiciones de temporada de sequía, presentándose valores entre $27^{\circ} \mathrm{C}$ y $28^{\circ} \mathrm{C}$. El mayor valor de temperatura, de $34,5^{\circ} \mathrm{C}$, se registró en el arroyo Cañafistula, en el punto 2 de muestra temporada de lluviosa. Lo anterior, se debe a que el oxígeno es menos soluble en agua caliente que en agua fría, por lo que la velocidad de las reacciones químicas sumado a la reducción del oxígeno, incide negativamente en las aguas residuales, aumentando los niveles de contaminación (Ornés y Chacón, 2009).

Turbidez. El intervalo de medición de la turbidez en otros países varía entre 0.05 a 5 UNT, tomán-

Figura 1. Comparativo de temperatura del arroyo en temporadas seca y lluviosa

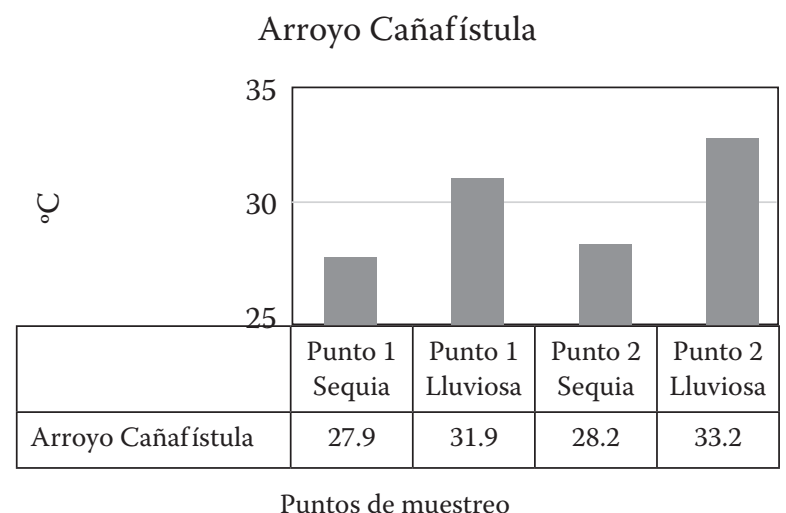

Fuente: elaboración propia dose como valor de referencia máximo la medida de 5 UNT. En Colombia la bibliografía consultada afirma que un valor 2 UNT se considera aceptable para el agua de consumo humano (potable). Los valores encontrados se expresan en UNT (Unidad Nefelométrica de Turbidez). No obstante, debido a la inexistencia de mediciones anteriores en el presente estudio no podrían establecerse patrones comparativos con datos de estudios preexistentes.

Se encontró que los niveles de turbidez más altos se presentaron durante la temporada lluviosa, debido a la dinámica natural de arrastre producto a la precipitación. Los niveles altos de turbiedad afectan el color de las muestras de agua, la temperatura y permiten inferir la presencia de materia orgánica e inorgánica que indirectamente ejerce un consumo del oxígeno presente en el agua (Ortega, Saldarriaga, García \& Wilches, 2010).

Conductividad. Los valores asociados a este parámetro se expresan en $\mathrm{uS} / \mathrm{cm}$. Reflejan la mineralización y la presencia de sales debido a vertimientos domésticos de asentamientos humanos. También se encontró que la conductividad es mayor durante la temporada seca y disminuye en temporada lluviosa, coincidiendo con lo planteado por Rodríguez (2002).

Así mismo, teniendo en cuenta lo expresado por Goyenola (2007), cada cuerpo de agua tiene un

Figura 2. Comparativo de turbiedad de los arroyos en temporadas seca y lluviosa

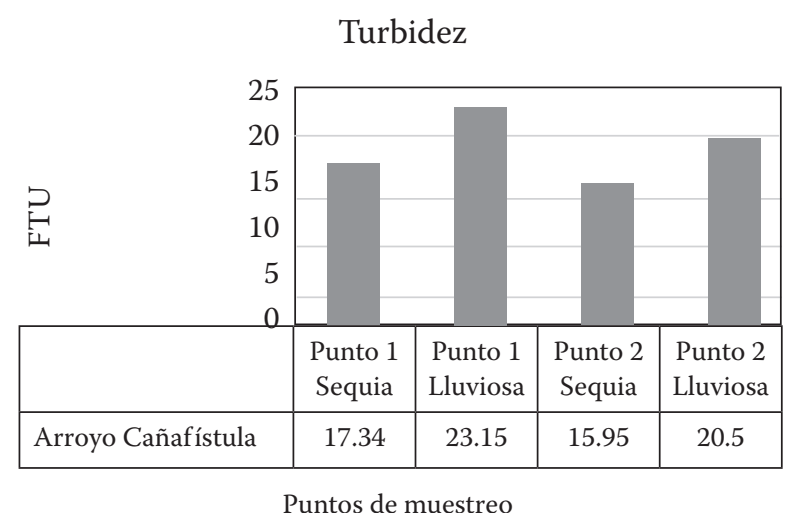

Fuente: elaboración propia 
Figura 3. Comparativo de conductividad de los arroyos en temporadas seca y lluviosa.

Conductividad

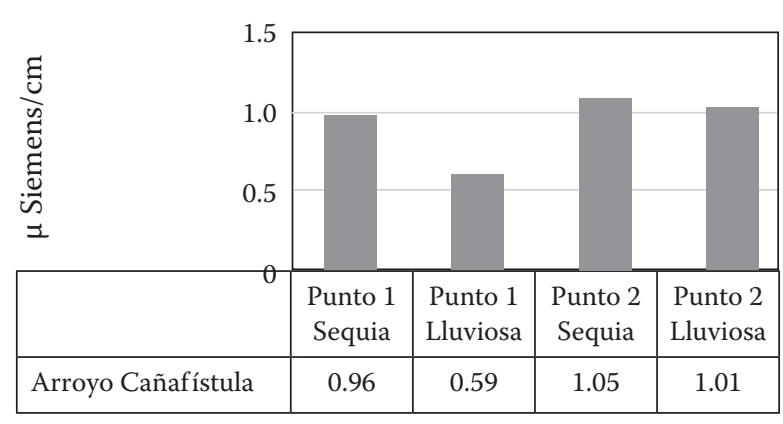

Puntos de muestreo

Fuente: elaboración propia

rango relativamente constante de conductividad, que una vez conocido, puede ser utilizado como línea de base para comparaciones con otras determinaciones puntuales, por lo que cambios significativos alcanzan ser indicadores de eventos puntuales de contaminación por aguas residuales debido al aumento de la concentración de Cl-, NO3 y SO4-2, u otros iones. Sin embargo, esta comparación no puede realizarse debido a la inexistencia de valores previos que permitan identificar variaciones significativas.

En cuanto a la relación de la conductividad con otros parámetros, puede afirmarse que la conductividad es directamente proporcional a la concentración de sólidos totales disueltos, por lo tanto cuanto mayor sea dicha concentración, mayor será la conductividad. Así mismo, la conductividad aumenta a medida que la temperatura aumenta, debido a que el transporte iónico disminuye ante temperaturas elevadas. (Hanna Instrument, 2017)

Sólidos totales disueltos. El Manual de Ecología Microbiana, publicado por Universidad de Puerto Rico (2002) considera esta característica física como la más importante, definiéndola como la suma de los minerales, sales, metales, cationes o aniones disueltos en el agua. Esto incluye cualquier elemento presente en el agua. Es evidente que los sólidos totales afectan la calidad de las
Figura 4. Comparativo de sólidos totales disueltos de los arroyos en temporadas seca y lluviosa

Arroyo Cañafístula

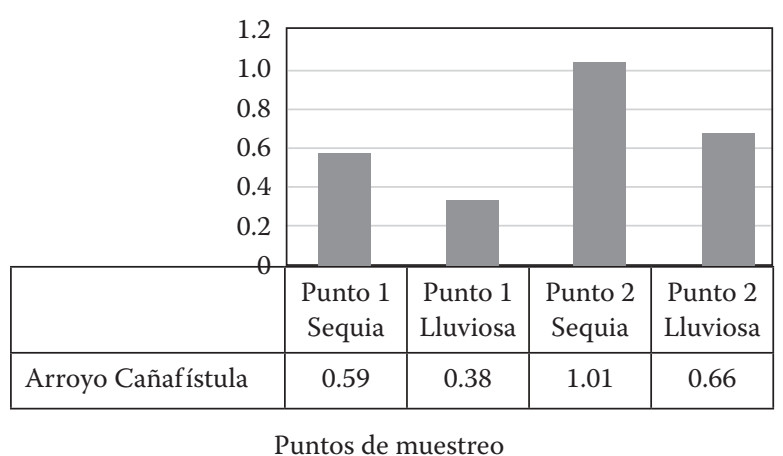

Fuente: elaboración propia

fuentes de agua, no sólo en su aspecto físico, sino también el químico al ocasionar reacciones fisiológicas adversas en las personas que la consumen. No se dispone de datos fiables sobre posibles efectos para la salud asociados a la ingestión de SDT presentes en el agua de consumo y no se propone ningún valor de referencia basado en efectos sobre la salud

Las aguas residuales, por lo general se caracterizan por la cantidad de residuos que arrastran, sin embargo, cabe anotar que en Colombia la Resolución 0631 del 2015, no manifiesta un valor límite tal como si lo hacen otros países, como es el caso de Estados Unidos, donde la Agencia de Protección Ambiental (EPA), en sus estándares para el agua potable estableció una concentración límite de 500 $\mathrm{mg} / \mathrm{L}$ de STD, teniendo en cuenta que la calidad del líquido para ser considerada buena debe estar por debajo de $600 \mathrm{mg} / \mathrm{L}$, cuando el valor supera los $1000 \mathrm{mg} / \mathrm{L}$ la palatabilidad del fluido disminuye significativa y progresivamente.

En los resultados obtenidos se observa que el Arroyo Cañafístula, posee nivel bajo de contaminación por este indicador.

Parámetros Químicos: Los parámetros químicos que se determinaron fueron: Demanda Química de Oxígeno (DQO), Demanda Biológica de Oxígeno $\left(\mathrm{DBO}_{5}\right), \mathrm{pH}$, Salinidad, Oxígeno disuelto 
y Potencial de óxido reducción, encontrándose los siguientes resultados:

Demanda Química de Oxígeno (DQO). Es un parámetro que mide la cantidad de sustancias susceptibles de ser oxidadas por medios químicos que hay disueltas o en suspensión en una muestra líquida. Se utiliza para medir el grado de contaminación y se expresa en miligramos de oxígeno diatómico por litro $\left(\mathrm{mg} \mathrm{O}_{2} / \mathrm{L}\right)$. Aunque este método pretende medir principalmente la concentración de materia orgánica, sufre interferencias por la presencia de sustancias inorgánicas susceptibles de ser oxidadas (sulfuros, sulfitos, yoduros), que también se reflejan en la medida.

Las concentraciones de DQO registradas para cuerpos de agua superficiales del arroyo Cañafístula se encontraron entre $36 \mathrm{mg} / \mathrm{L}$ y $86 \mathrm{mg} / \mathrm{L}$ en condiciones de tiempo de sequía y lluvia, respectivamente. Los valores y relaciones reportadas podrían sugerir una escasa presencia de descargas industriales de compuestos que generan demanda oxígeno por materia orgánica de difícil degradación tales como surfactantes fosfatados, cadenas poliméricas complejas como plásticos y siliconas y compuestos orgánicos, entre otros (Henze, 1997).

Puede observarse que al medir el nivel de DQO este arrojó valores bajos (entre 36,92 y 86,6), sin

Figura 5. Comparativo de Demanda Química de Oxígeno en los arroyos en temporadas seca y lluviosa

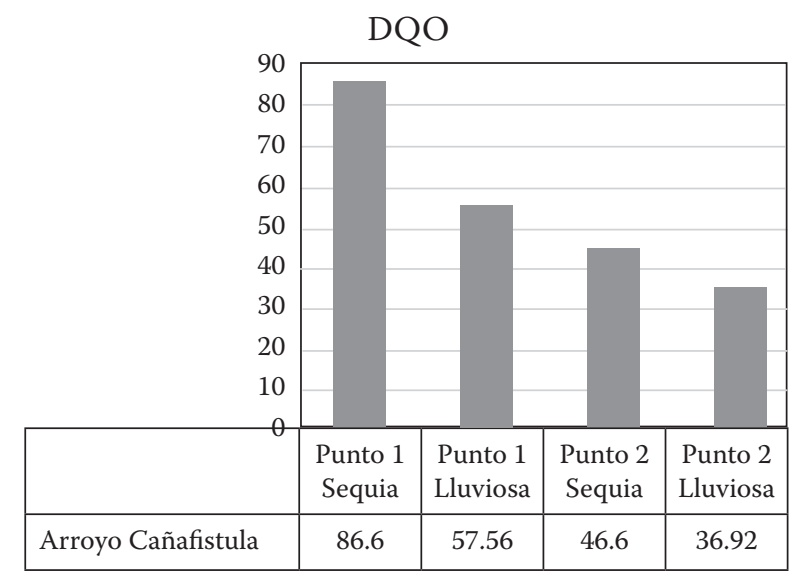

Puntos de muestreo

Fuente: elaboración propia embargo no significa que se encuentra libre de contaminación, ya que estos resultados sugieren una escasa presencia de descargas industriales, como se evidenció en estudios similares realizados en los caños Las Compañías, La Ahuyama y Arriba y el caño de Soledad, según Mefcalt y Edy (1985).

Demanda Biológica de Oxígeno. Respecto a los valores obtenidos para la $\mathrm{DBO} 5$ en las diferentes estaciones de monitoreo, éstos se encontraron entre $4 \mathrm{mg} / \mathrm{L}$ y $47 \mathrm{mg} / \mathrm{L}$, obteniéndose los mayores valores en condiciones de tiempo lluvia los cuales presentan un valor promedio de $8,95 \mathrm{mg} / \mathrm{L}$.

Este parámetro indispensable cuando se necesita determinar el estado o la calidad del agua. Según Duran y Lara (1994) este análisis se realiza midiendo el oxígeno que consume una población microbiana en condiciones que por lo general favorecen el desarrollo de los microorganismos.

El valor de DQO osciló entre 57-86 mg/L. $\mathrm{La} \mathrm{DBO}_{5}$ es otro parámetro indicador de materia orgánica que evalúa la cantidad de oxígeno requerida para oxidar la materia orgánica biodegradable presente. Como se observa, la relación del comportamiento entre la DQO y la $\mathrm{DBO}_{5}$ es directa, con un coeficiente de correlación de Pearson de 0,1095, lo que indica una correlación débil entre ambos parámetros (SEQ1).

Figura 6. Comparativo de $\mathrm{DBO}_{5}$ en el arroyo en temporadas seca y lluviosa

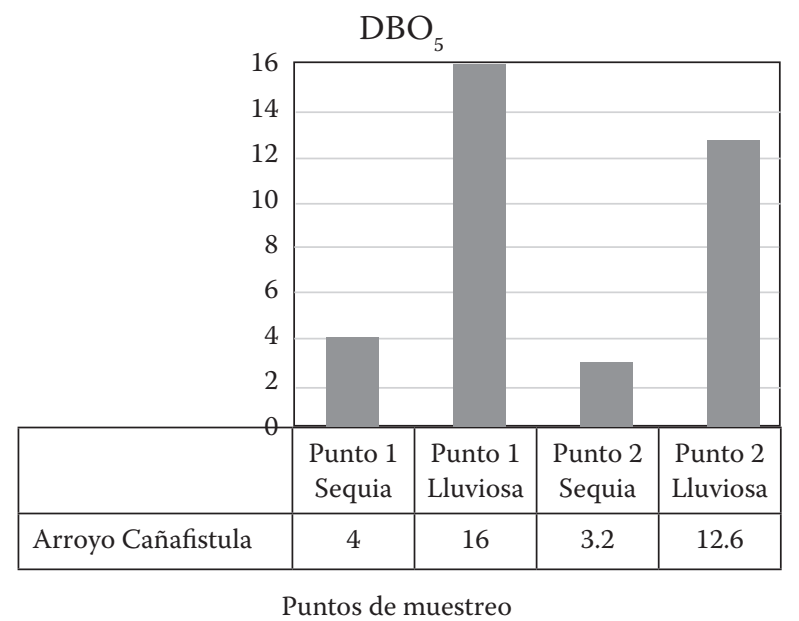

Fuente: elaboración propia 
Cuando la relación $\mathrm{DBO}_{5} / \mathrm{DQO}>0,6$ predomina materia orgánica biodegradable y si es $<0,2$ no es biodegradable. La relación de $\mathrm{DBO}_{5} / \mathrm{DQO}$ arrojada en esta investigación fue 0,3 por lo que puede considerarse la existencia en el agua de materia orgánica moderadamente biodegradable. Esto sugiere que pueden ser aplicados sistemas de tratamientos químico-físicos (primarios) y biológicos (secundarios). Los sistemas de tratamientos primarios facilitan la eliminación de sólidos totales y la oxidación de compuestos vía reacciones químicas en lapsos de tiempo pequeños y condiciona la acción posterior de los organismos usados en los tratamientos biológicos.

Salinidad. Los resultados obtenidos para la salinidad en las diferentes estaciones de monitoreo registraron valores que oscilan entre $0,20 \mathrm{mg} / \mathrm{L}$ y 0,63 mg/L, obteniéndose los rangos más elevados en temporada de sequía. Situación acorde a lo expuesto por Mujeriego (1990), el cual denota que el uso doméstico del agua aumenta el contenido de sales, por lo general contiene el agua de abastecimiento, que se ubica entre los 150-400 $\mathrm{mg} / \mathrm{L}$ y que al ser vertidas en la fuente hídrica ocasionaría aumento en los niveles de contaminación de éstas.

Figura 7. Comparativo de Salinidad en el arroyo en temporadas seca y lluviosa

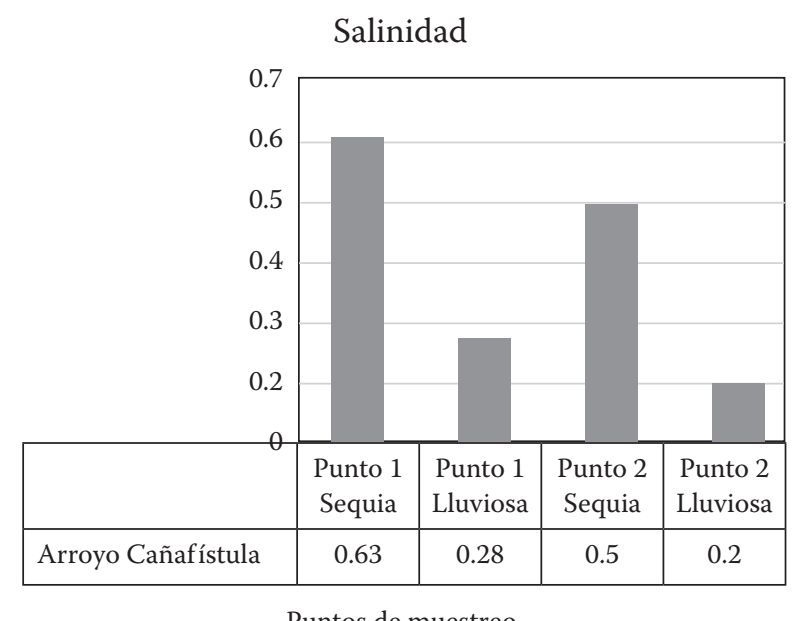

Fuente: elaboración propia
pH. Respecto a los valores obtenidos para el pH en las diferentes estaciones de monitoreo, éstos se encontraron entre 6 a 9 , obteniéndose los mayores valores en temporada de lluvia, siendo el arroyo Pica Pica el cuerpo de agua en el que se registran las mayores concentraciones, con valores entre 8,48 y 8,96 ; mientras que, en condiciones invernales, por efecto de la dilución, las concentraciones obtenidas oscilaron entre 5,9 y 6,3 .

Los resultados obtenidos de las muestras tomadas a los arroyos Cañafístula y Pica Pica, se muestran dentro de los niveles establecidos en el Decreto 1076 de 2015 (6 a 9 unidades), sin embargo los límites en temporada lluviosa del arroyo Pica Pica se acercan al límite permitido, teniendo en cuenta la influencia del arrastre de sustancias y materiales contaminantes que recoge dentro de la cabecera urbana en su cauce y que acidifican las aguas residuales, se asume que no origina afectación significativa al medio ambiente; por su parte, Mujeriego (1990), explica que el pH del agua residual afecta a la solubilidad de los metales así como a la alcalinidad del suelo. Por esta razón, el intervalo normal para el $\mathrm{pH}$ del agua residual se sitúa entre los valores de 6 a 9 unidades.

Figura 8. Comparativo de $\mathrm{DBO}_{5}$ en el arroyo en temporadas seca y lluviosa

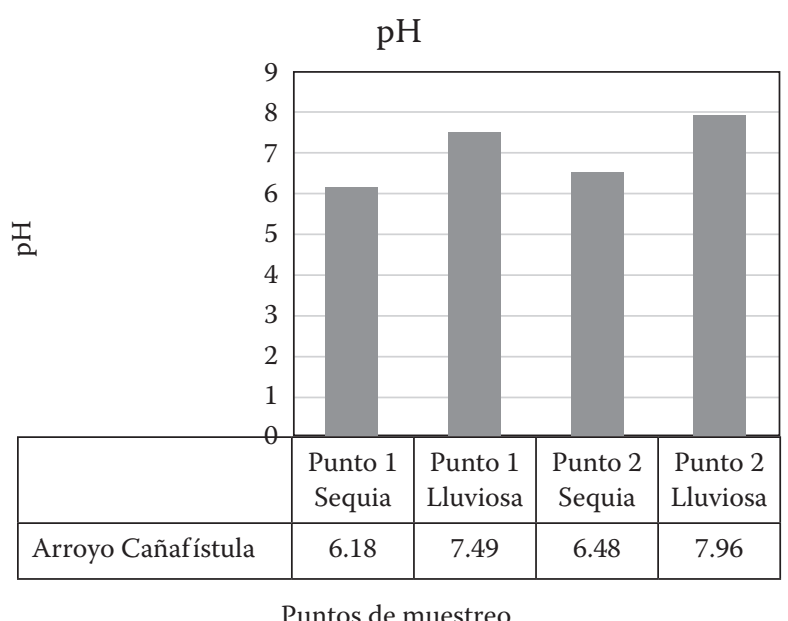

Fuente: elaboración propia 
Oxígeno disuelto. En cuanto a los niveles de oxígeno disuelto en los cuerpos de agua monitoreados, estos presentaron valores entre $3,1 \mathrm{mg} / \mathrm{L}$ y $6,18 \mathrm{mg} / \mathrm{L}$ para el arroyo Cañafístula en condición de tiempo seco, en condiciones invernales los valores oscilaron 4,69 y 7,20 mg/L. Siendo uno de los índices más representativos del grado de contaminación de una corriente, el nivel de oxígeno disuelto mantiene niveles superiores a los $3 \mathrm{mg} / \mathrm{L}$ en los diferentes puntos analizados.

Es importante considerar que las principales fuentes de oxígeno en un cuerpo de agua corresponden a: la difusión del gas desde la atmósfera; y a la producción fotosintética de las plantas acuáticas. No obstante, lo anterior, las descargas en la zona y los efluentes residuales no tratados vertidos por los municipios, sumado a la presencia de macrófitas, pueden sugerir la afectación de la concentración de oxígeno disuelto en la columna de agua por efecto de los procesos de fotosíntesis y respiración (Mathers et al., 2002).

Al contrastar estos niveles, se observa que los resultados obtenidos en el arroyo Cañafístula en la temporada de sequía denotan valores bajos, entendiendo que la situación se origina por la poca precipitación y la alta temperatura, ocasionando un volumen limitado de agua a diferencia a la época

Figura 9. Comparativo de niveles de oxígeno disuelto del arroyo en temporadas seca y lluviosa

\section{Arroyo Cañafístula}

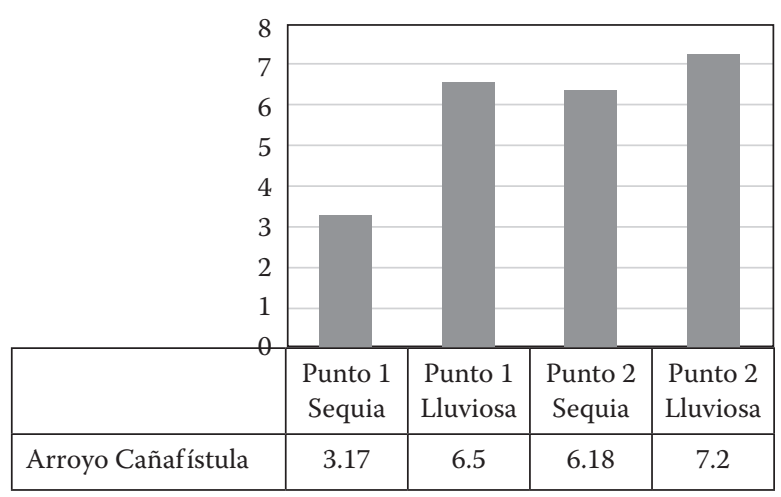

Puntos de muestreo

Fuente: elaboración propia de lluvia; sin embargo, al realizar una comparación entre los parámetros analizados se pudo observar que la Demanda Bioquímica de Oxígeno $\left(\mathrm{DBO}_{5}\right)$ genera un impacto negativo en el ecosistema ya que reduce la cantidad de oxígeno disponible en el agua. El informe del Ministerio de Salud (2012) estima que en el país la carga total de DBO fue de 729.383 toneladas, siendo el sector doméstico el que tuvo una mayor aportación (65\%), frente a otros sectores económicos que tienen incidencia en este indicador; no obstante, la correlación existente entre el $\mathrm{DBO}_{5}$ y el OD fue débil (SEQ5).

Potencial de óxido reducción. Normalmente, las reacciones redox vienen acompañadas de cambios de $\mathrm{pH}$ en el medio; diversas investigaciones de la Universidad de Puerto Rico en 2012 han determinado que ambientes de agua dulce en equilibrio con el oxígeno atmosférico, tienen valores de potencial de óxido reducción alrededor de $(0.8 \mathrm{mV})$, por lo que al compararlos con los datos obtenidos en los puntos de muestreo de ambos arroyos se observa que los valores probablemente estuvieron influenciados por las condiciones de óxido-reducción que predominan en el sistema debido a la degradación del material orgánico particulado y la naturaleza del terreno, convirtiéndose en un indicador de contaminación latente.

Figura 10. Comparativo del Potencial de óxido reducción en los arroyos en temporadas seca y lluviosa

Arroyo Cañafístula

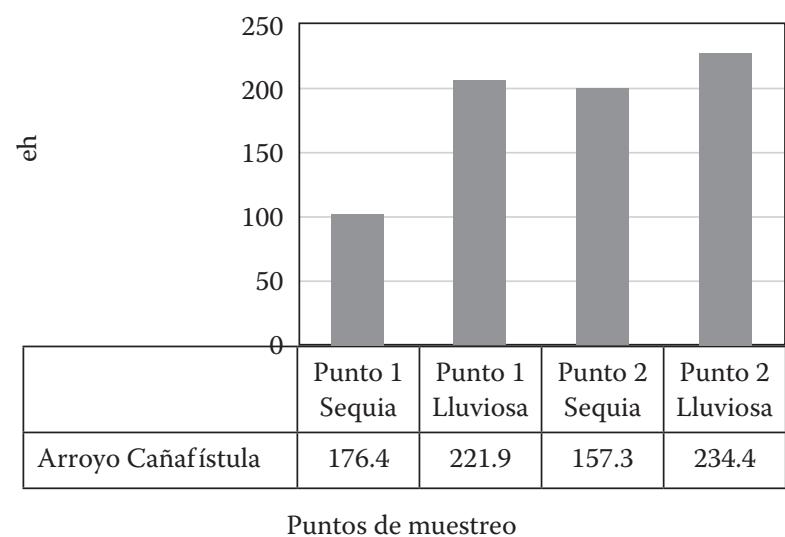

Fuente: elaboración propia 


\section{Conclusiones}

Los estudios fisico-químicos llevados a cabo en el arroyo Cañafístula, arrojaron valores que se encuentran dentro de los límites establecidos en la normatividad vigente, Decreto 1076 de 2015. No obstante, se encontró que éste recibe aguas residuales debido a la carencia de alcantarillado municipal y al escaso conocimiento de los habitantes para el manejo de las mismas. Se infiere que cada una de las viviendas anexadas al arroyo son fuentes puntuales de contaminación, aunque no se apreciaron resultados elevados en los parámetros evaluados.

Las fuentes puntuales de contaminación de los cuerpos de agua son en primera instancia resultado de la falta de alcantarillado en el municipio. Esta situación, obliga a los residentes a hacer uso de pozas sépticas de las cuales desconocen el tamaño o manifestaron no haberles hecho nunca mantenimiento, infiriéndose que existe filtración al suelo.

Por otra parte, las aguas domésticas en su mayoría no son reutilizadas, sino que son vertidas al patio de los hogares con salida hacia la calle. Al igual ocurre con el agua de los baños que se ubican al exterior de la vivienda, el agua corre hacia la calle a través de canales creados para tal fin y de esta manera son vertidos al arroyo. A lo largo del mismo se encontraron viviendas con un número considerable de habitantes, los cuales en algunas ocasiones se ven abocados a realizar sus necesidades o su higiene personal al aire libre, por carecer de alcantarillado, contaminado el cuerpo de agua de forma: directa, al ser vertidas en su cauce; ó indirecta, a través del arrastre realizado por las lluvias.

Las características del agua del arroyo Cañafístula no difieren de las de otros cuerpos de agua pertenecientes a la cuenca de los humedales del río Magdalena. No obstante, aunque su agua sea más clara debido a la filtración natural de la tierra y las plantas presentes en su curso, denota la presencia de coliformes fecales y totales, razón por la cual no se recomienda para consumo humano. En este mismo sentido, se encontró que los datos obtenidos de las estaciones de muestreo, los resultados indicaron menores valores de contaminación en los orígenes del arroyo que en sectores más alejados de este, situación dada por encontrarse fuera de zonas habitadas, mientras que los sectores del cauce con mayor presencia de habitantes presentaban un índice más elevado de contaminación.

Es de anotar que los resultados con relación a la contaminación obtenidos se encuentran por debajo de los límites establecidos en la normatividad nacional vigente. Sin embargo, se hace necesario tomar las acciones pertinentes para mejorar estos cuerpos de agua, debido al beneficio que le ofrece a la población rural y urbana del municipio. Por ello es perentorio la toma de acciones tendientes a la recuperación, conservación y cuidado del mismo, sin dejar a un lado que el arroyo es afluente de una de las cuencas hidrográficas que vierte sus aguas al río Magdalena y su impacto ecológico podría ser mayor si no se toman las medidas necesarias, afectando directamente a los habitantes en los aspectos sociales, económicos y principalmente en la calidad de vida que esta fuente hídrica le puede brindar.

\section{Referencias Bibliográficas}

Alcaldía de Polonuevo. (2016). Plan de Desarrollo Municipal de Polonuevo 2016-2019. Recuperado de: http://polonuevo-atlantico.gov.co/apc-aafiles/373834343233633739353361336 43966/0. plan-de-desarrollo-territorial-versin-final.pdf

APHA (2005). Standard methods for the examination of water and wastewater, 21st edn. American Public Health Association, Washington, DC. Recuperado de: https://www.mwa.co.th/download/file_upload/SMWW_1000-3000.pdf

Duran, D. y Lara, A. (1994). Convivir en la tierra. Buenos Aires, Argentina: Lugar Editorial.

Espigares García, M. y Pérez López, JA. (1985). Aspectos sanitarios del estudio de las aguas. Granada, España. Universidad de Granada. 
FAO (2015). Glosario. Organización de las Naciones Unidas para la Alimentación y la Agricultura. Recuperado de: http://www.fao.org/nr/water/ aquastat/data/glossary/search.html

Goyenola, G. (2007). Guía para la utilización de las Valijas Viajeras. Red de Monitoreo Ambiental Participativo de Sistemas Acuáticos. RED MAPSA. [Consultado el 2, febrero, 2017] Recuperado de: http://imasd.fcien.edu.uy/difusion/ educamb/propuestas/red/curso_2007/cartillas/ tematicas/Conductividad.pdf

Hanna Instruments (2017). Conductividad eléctrica y temperatura [en línea] Recuperado de: http://www.hannainst.es/blog/conductividadelectrica-y-temperatura/

Henze, M. (1997). Waste design for households with respect to water, organics and nutrients. Wat. Sci. Tech.35(9), pp. 113-120.

Hidalgo, M; Mejía, E. (2010). Diagnóstico de la contaminación por aguas residuales domésticas, cuenca baja de la quebrada la macana, San Antonio de Prado. Municipio de Medellín (Tesis de especialización). Universidad de Antioquia, Medellín, Colombia. Recuperado de: http:// www.juntadeandalucia.es/medioambiente/site/ portalweb/menuitem.7e1cf46ddf59bb227a9eb e205510e1ca/?vgnextoid=19aebdaa541a5210 VgnVCM1000001325e50aRCRDyvgnextchan nel=48ace3dbc95f4310VgnVCM2000000624e 50aRCRD

Mathers, C.; Bernard, C.; Moesgaard Iburg, K.; Inoue, M.; Ma Fat, D.; Shibuya, K.; Stein, C.; Tomijima, N. \& Xu, H. (2002). Global Burden of Disease in 2002: data sources, methods and results. World Health Organization. Recuperado de: http://www.who.int/healthinfo/paper54.pdf

Ministerio de Agricultura (2011). Proyecto: desarrollo de capacidades en el uso seguro de aguas residuales para agricultura. Recuperado de: http://www.ais.unwater.org/ais/pluginfile.php/ $356 /$ mod_page/content/128/Colombia_ Informe\%20Nacional.pdf
Ministerio de Ambiente (2015). Decreto Único Reglamentario del Sector Ambiente y Desarrollo Sostenible. Decreto 1076 del 26 de mayo de 2015. Recuperado de: http://www.minambiente.gov.co/index.php/component/content/ article/81-normativa/2093-plantilla-areasplaneacion-y-seguimiento-30

Mujeriego, R. (1990). Manual Práctico de Riego con Agua Residual Municipal Regenerada. Barcelona, España. Ediciones de la Universitat Politécnica de Catalunya. Recuperado de: http:// mie.esab.upc.es/arr/T23E.htm

Núñez,A. (2015). Caracterización dela problemática de las aguas residuales en Ixmiquilpan Hidalgo. México (Tesis de Maestría) Universidad Autónoma Metropolitana. México. Recuperado de: http://dcsh.izt.uam.mx/licenciaturas/geografia humana/wp-content/uploads/2015/09/ Tesina-Ana-Laura-Nu\%C3\%B1ez-2015.pdf

OCDE (2014). Panorama de la educación. Indicadores dela OCDE. Instituto Nacional deEvaluación Educativa. Recuperado de: http://www. mecd.gob.es/dctm/inee/indicadores-educati vos/panorama2014/panorama-de-la-educacion2014informe-espanol-05-sep-.pdf?documentId $=0901 \mathrm{e} 72 \mathrm{~b} 81 \mathrm{a} 722 \mathrm{ac}$

Ornés, S., y Chacón, R. M. (2009). La gestión de riesgo ambiental como eje de la planificación urbana e indicador de desarrollo local. Paper, Universidad Simón Bolívar. Caracas, Venezuela. Departamento de Planificación Urbana. Recuperado de: http://prof.usb.ve/sornes/doc/ Articulo-CISDA-SOV2009.pdf

Ortega, L.C.; Saldarriaga, G.; García, M.; Wilches, H. (2010). Capítulo 6: Calidad del agua superficial en Colombia. En: Estudio Nacional del agua 2010. IDEAM. Recuperado de: https:// datoscede.uniandes.edu.co/anexo/materialrelacionado/d16_Informe.pdf

PNUMA (2004). Lineamientos para el tratamiento de aguas residuales. Recuperado de: https://esa. un.org/iys/docs/san_lib_docs/lineamientos_ sobre_el_manejo_spanish.pdf 
PNUMA (2016). A Snapshot of the World's Water Quality: Towards a global assessment. Executive Summaries. Recuperado de: http:// www.wwqa-documentation.info/assets/sum spanish_unep_wwqa_report_web2.pdf

Ramírez Hernández, O. (2015). Identificación de problemáticas ambientales en Colombia a partir de la percepción social de estudiantes universitarios localizados en diferentes zonas del país. Revista internacional de contaminación ambiental, 31(3), 293-310. Recuperado en 29 de mayo de 2018, de http://www.scielo.org. $\mathrm{mx} / \mathrm{scielo}$.php? script $=$ sci_arttext\&pid=S018849992015000300009\&lng=es\&tlng=es.

Rodríguez, E. (2002). Las lagunas continentales de Tabasco. Tabasco. México. Universidad Juárez Autónoma de Tabasco.

Rosedo, N. (2014). Impacto ambiental y plan de manejo ambiental en la descarga de aguas residuales generada por la operación y mantenimiento de la planta de tratamiento del barrio la primavera de la parroquia yana- yacu cantón quero provincia de Tungurahua (Tesis de maestría) Universidad Técnica de Ambato, Ecuador. Recuperado de: http:// repo.uta.edu.ec/bitstream/123456789/6798/1/ tesis-007\%20Maestr\%C3\%ADa\%20en\%20 Agroecolog\%C3\%ADa\%20y\%20Ambiente\%20 -\%20CD\%20221.pdf

UNESCO (2016). Informe de las Naciones Unidas sobre el Desarrollo de los Recursos Hídricos en el Mundo. 2016. Recuperado de: http://unesdoc. unesco.org/images/0024/002441/244103s.pdf

Universidad de la Guajira (2016). Protocolo de Determinación de Demanda Bioquímica de Oxígeno. Recuperado de: http://sigug.uni gua jira.edu.co/sigug/pdf/PROTOCOLOS/ GL-PL-25.\%20PROTOCOLO\%20DBO\%205. pdf

Universidad de Puerto Rico (2002). Manual de ecología microbiana. Capítulo 2: Parámetros físico-químicos: Sólidos disueltos totales. Recuperado el 2/02/17 de: http://www.uprm.edu/ biology/profs/massol/manual/p2-tds.pdf 